\title{
Post traumatic stress disorder: undiagnosed cases in a tertiary inpatient setting
}

\author{
M Van Zyl, PP Oosthuizen, S Seedat \\ Department of Psychiatry, Faculty of Health Sciences, University of Stellenbosch, Stellenbosch, South Africa
}

\begin{abstract}
Objective: Post traumatic stress disorder (PTSD) is a common, debilitating anxiety disorder characterized by emotional and physical symptoms that may occur after exposure to a severely traumatic event. Since it occurs commonly as a comorbid diagnosis with other mood- and anxiety disorders, we postulated that this disorder may be under- diagnosed in therapeutic wards where anxiety and mood disorders are treated. The study thus sought to determine the prevalence of undiagnosed PTSD in an inpatient population, and to compare the demographic details and comorbid diagnoses of subjects with and without PTSD. Method: The Clinician-administered PTSD Scale for DSM-IV (CAPS) was administered to 40 subjects who were inpatients in a therapeutic ward of a large psychiatric hospital and who had never had a diagnosis of PTSD before. Results: 16 (40\%) subjects met the DSM-IV criteria for PTSD. We did not find significant clinical differentiating factors between subjects with and without PTSD; however subjects with PTSD were more likely to use cannabis. Conclusions: PTSD remains undiagnosed in many patients admitted to therapeutic units.
\end{abstract}

Key words: Stress Disorders, Post-Traumatic; Diagnosis; Inpatients;

Received: $26-06-2007$

Accepted: 17-09-2007

\section{Introduction}

Posttraumatic stress disorder (PTSD) may develop after a person experiences, sees or hears about a traumatic event. It is one of the most common anxiety disorders in the general population, with a lifetime prevalence of 5\%-10\%, almost half of that of major depression. ${ }^{1}$ The precipitating event is of a serious nature, and involves actual or threatened death, or serious injury to self or others to which the person responds with feelings of fear, helplessness or horror. The DSM-IV furthermore requires that the patient also has symptoms in each of the following 3 symptom clusters: re-experience, avoidance and increased arousal. ${ }^{2}$

In general, approximately a quarter of people witnessing a traumatic event will develop PTSD. ${ }^{3}$ Risk factors that have been

\section{Correspondence:}

Prof PP Oosthuizen

Department of Psychiatry, University of Stellenbosch,

PO Box 19063, Tygerberg, 7505, South Africa

email: pieto@sun.ac.za suggested include: childhood abuse, previous psychiatric history and family history of psychiatric illness. ${ }^{4}$ It is widely accepted that a personal predisposition also makes a major contribution to the development of the disorder. ${ }^{5}$ In terms of the traumatic event itself, it seems that the severity, rather than the type of trauma tends to be of significance as a risk factor, and may also be related to the chronicity and severity of PTSD. ${ }^{6}$ Even though PTSD leads to significant distress and may increase the use of healthcare resources ${ }^{7}$, it has been described as a "hidden diagnosis". 8 Patients may be unlikely to endorse traumatic experiences when they present with physical or psychiatric complaints or may not think them to be relevant to their current condition.

PTSD commonly co-occurs with other disorders. The National Comorbidity Study in the USA showed that 59\% of men with PTSD and 44\% of women with PTSD met the criteria for 3 or more psychiatric diagnoses. ${ }^{9}$ The presence of other, co-morbid psychiatric disorders therefore seems to be the rule rather than the exception. The most common co-morbid disorders include Major Depressive Disorder and other mood disorders, other anxiety disorders and substance abuse 
disorders. ${ }^{9}$ Treatment options for PTSD include both psychological and pharmacological interventions. Although psychological interventions aimed at preventing PTSD have been ineffective, trauma focused cognitive-behavioural therapy consisting of combinations of cognitive reprocessing, eye-movement desensitization training, exposure and cognitive restructuring has been successful in the treatment of this disorder. ${ }^{10}$ Pharmacological treatments include most of the SSRI's as well as mirtazapine and phenelzine. Some studies have suggested that the addition of atypical antipsychotics may also be a treatment option. ${ }^{11}$

Although PTSD is highly prevalent among populations of inpatients admitted for serious mental illness, it is rarely reflected in the primary or secondary diagnosis, and thus rarely becomes the focus of treatment. ${ }^{12}$ Because the incidence of trauma is so disturbingly high in our society, leading authors in the field of PTSD have recommended that all psychiatric interviews, but also interviews with patients with unexplained somatic complaints, should include routine screening for trauma. ${ }^{13}$ It is suggested that clinicians should specifically ask about traumatic events as many individuals will not volunteer this information because they either find it painful to talk about, or think that it might not have bearing on the present complaints. It is vital that the correct diagnosis be made, and therefore important that the trauma history be thoroughly assessed.

The aims of this study were twofold:

1. To determine the prevalence of undiagnosed PTSD in a cohort of subjects admitted to a therapeutic ward at a large psychiatric hospital.

2. To compare the demographic details and comorbid diagnoses of subjects with and without PTSD.

\section{Method \\ Subjects}

This study was conducted over a six month period at Neuroclinic C, Stikland Hospital in Bellville, Cape Town. Neuroclinic $\mathrm{C}$ is an inpatient, therapeutic unit for patients with mood and anxiety disorders. Subjects were randomly recruited from the inpatient population of the unit using a computer generated randomization table. The current study represents a cross-sectional assessment of patients admitted for anxiety and mood disorders. The evaluation could be performed at any stage during the inpatient admission period, provided that a diagnosis and treatment plan had been formulated by the treating team. Assessments for the purposes of the study were therefore not performed for at least two days after admission. All participants gave written, informed consent before undergoing any study-related procedures. The study was approved by the institutional review board of the University of Stellenbosch.

Inclusion criteria

1. Male or female inpatients.

2. Aged between 18 and 55 years (extremes included).

3. DSM-IV diagnosis of Mood Disorder or Anxiety Disorder (excluding PTSD).

4. Current hospitalization in inpatient, therapeutic ward.

5. Subject had signed the informed consent form, according to the regulations of the ethics committee.
Exclusion criteria

Subjects meeting one or more of the following criteria could not be entered:

1. Evidence of alcohol or drug dependence in the last month before screening.

2. Serious physical illness.

3. Mental retardation.

4. History of psychotic episode.

5. Subjects with a known diagnosis of PTSD.

\section{Assessment}

The following scales were administered:

- CAPS (Clinician-administered PTSD scale for DSM-IV). ${ }^{14}$

- SCID (Structural Clinical Interviews for DSM-IV Disorders). ${ }^{15}$

- Demographic Information and Substance Use questionnaire

\section{Statistical analysis}

Statistical analysis was performed with the computer software programme Statistica (Statsoft, Inc.) Descriptive data are presented (where appropriate) as mean ( \pm standard deviation). Where categorical variables were compared, we used the chi-square test or Fisher's exact test, depending on expected frequencies. Between-group comparisons were done with Student's T-test or the Mann-Whitney U test, depending on the distribution of the data. A significance level of 0.05 was used throughout.

\section{Results}

40 subjects were included in the study of whom 33 (82.5\%) were female. The mean age of the group was 35.65 years ( \pm 9.28 years). 19 (47.5\%) of the subjects were employed at the time of interview. Those who were employed were all in fulltime employment. Subjects with undiagnosed PTSD were not more or less likely to be employed than subjects without PTSD $\left(\chi^{2}=0.07 ; \mathrm{df}=1 ; \mathrm{p}=0.8\right)$.

\section{Ethnic distribution}

Twenty-two subjects (55\%) described themselves as being of mixed ethnic origin (55\%) with 17 (42.5\%) describing themselves as Caucasian and 1 (2.5\%) as Black African. Subjects had a mean number of 1.9 admissions ( \pm 2.2 ) when they consented to the study.

\section{Prevalence of PTSD}

After administering the CAPS, it was found that 16 (40\%) subjects met the DSM-IV-TR criteria for PTSD. It must be noted that out of a total of 293 patients admitted to this ward during the time the study was undertaken 30 patients had a discharge diagnosis of PTSD (this includes the 16 patients diagnosed with PTSD as a direct result of the study). Although there was a very large percentage difference between the genders in the prevalence of undiagnosed PTSD (14.3\% male vs. $45.5 \%$ female) this did not reach statistical significance ( $p=0.21$, fisher's exact test, two tailed), probably due to the study being underpowered. The ethnic groups did not differ significantly in the risk of having PTSD ( $p=0.34$, Fisher's exact test, two tailed). We did not find any significant difference between the groups in the lifetime use of alcohol or illicit drugs, excluding cannabis ( $p=0.63$, Fisher's exact test, two-tailed). Eleven 
subjects (27.5\%) admitted to using cannabis. Subjects with PTSD were significantly more likely to have used cannabis ( $\mathrm{p}$ $=0.01$, Fisher's exact test, two tailed). Admission diagnoses for subjects with PTSD are listed in Table I. The causes of PTSD, as identified by the subjects, are presented in Tables II and III.

\begin{tabular}{|l|l|}
\hline \multicolumn{2}{|l|}{ Table I: Admission diagnoses for subjects with PTSD } \\
\hline Diagnosis & Percentage of subjects \\
\hline Dysthymic Disorder & $13 \%$ \\
Bipolar I Disorder & $13 \%$ \\
Bipolar II Disorder & $19 \%$ \\
Adjustment Disorder & $13 \%$ \\
Major Depressive Disorder & $44 \%$ \\
\hline
\end{tabular}

Table II: Traumatic events leading to PTSD (as identified by subjects.)

\begin{tabular}{|l|l|}
\hline Cause & Percentage of subjects \\
\hline Accident & $6 \%$ \\
Multiple causes & $44 \%$ \\
Sexual assault/abuse & $19 \%$ \\
Assault (other) & $31 \%$ \\
\hline
\end{tabular}

Table III: Traumatic events identified by subjects with multiple causes of PTSD

\begin{tabular}{|l|l|}
\hline $\begin{array}{l}\text { Subject } \\
\text { number }\end{array}$ & Traumatic events \\
\hline 1 & Motor vehicle accident; sexual assault, threatened with fire-arm \\
2 & Witnessed rape, victim of robbery \\
3 & Physical assault; rape \\
4 & Severe physical illness, rape \\
5 & Rape, witnessed death of spouse \\
6 & Assault, rape, motor vehicle accident \\
7 & Witness to a murder, witness to suicide \\
\hline
\end{tabular}

\section{Discussion}

PTSD is a debilitating anxiety disorder that can cause significant distress and lead to increased utilization of health care resources. ${ }^{7}$ However, the condition often goes unnoticed. The annual cost of anxiety disorders in the USA was estimated to be $\$ 63$ billion dollars in 1998, and the cost of PTSD related reduced productivity (based on work days lost and work cutback days) translates to a financial loss of more than $\$ 3$ billion annually. PTSD compares with major depression in the level of disability it imposes on individuals with the disorder.

There is evidence that PTSD prevents individuals from realizing their potential, either in educational attainment, marriage or career development. ${ }^{1}$ Secondary mental disorders (as discussed above) may also be seen as a consequence of PTSD. Individuals with PTSD are substantially more likely to develop other anxiety, mood and substance use disorders. PTSD also increases the risk of suicide ${ }^{16}$ and complicates the process of recovery from other disorders. ${ }^{17}$ Furthermore, it seems that the elevated risk of secondary disorders disappears with the remission of PTSD symptoms. ${ }^{18}$

Only a few studies have explored the epidemiology of
PTSD in South Africa. Seedat et al ${ }^{19}$ analyzed data from a sample of 2041 boys and girls from schools in Cape Town and Nairobi, and reported that $58 \%$ of the South African schoolchildren had witnessed trauma, and that $22,2 \%$ met the full-symptom criteria for PTSD. Carey et $\mathrm{al}^{20}$ evaluated the prevalence of trauma exposure and PTSD in a Xhosa-speaking township, and reported that $94 \%$ of the 201 participants had been exposed to trauma, and 19.9\% had current PTSD. Comorbidity was high and included depression (75\%), somatization (35\%), and panic disorder (25\%). Levels of trauma, PTSD and depression did not increase service use or dissatisfaction with services. ${ }^{20}$

The high percentage of subjects with undiagnosed PTSD confirmed our hypothesis that the disorder is often not recognized even in a tertiary, inpatient setting. Whereas the prevalence of PTSD in the study group was 40\%, only 5.5\% of the patients admitted to the neuroclinic - study participants excluded - attracted a diagnosis of PTSD. Due to the high comorbidity of PTSD and other disorders it would be expected that a higher percentage of patients would be diagnosed in hospital, even if the diagnosis had been missed by the referring professional. Why were so many patients not diagnosed and therefore not treated for this disorder? It can be postulated that the diagnosis of PTSD may be overlooked if the admitting physician does not specifically ask about traumatic events, as patients are unlikely to volunteer this information. However, this is not only true in our setting. The literature shows that PTSD is under-diagnosed throughout the spectrum of care - from primary to tertiary settings, and it appears to be a problem affecting patient care in first as well as third world countries. ${ }^{8}$

The co-morbidity of PTSD and substance abuse is well described in the literature. This was also true in our study when patients with and without PTSD were compared, patients with PTSD were significantly more likely to be users of cannabis. This was not the case with other substances, such as alcohol. It would appear that patients with undiagnosed PTSD use cannabis to self-medicate their PTSD symptoms, or alternatively that patients who are cannabis users are more prone to develop PTSD when exposed to trauma. This possibility implies another reason to diagnose PTSD early and correctly, as the long term dangers of cannabis use have been well described. ${ }^{21}$

The patients seen for this study were diagnosed with PTSD according to the CAPS, which uses the DSM IV criteria. The CAPS is a comprehensive, clinician rated questionnaire that covers the details of each symptom (e.g. frequency and intensity) and how this varied as time passed after the traumatic event was experienced. It remains the gold standard to measure PTSD symptom..$^{22}$ Due to the time-consuming nature of the CAPS, it is however not likely to be of practical use in a busy, clinical setting. Instruments such as the Short PTSD Rating Interview (SPRINT) were still under development when this study was undertaken 6 , and there is now evidence that the validity of this instrument (SPRINT) may be comparable to that of the CAPS. ${ }^{23}$

This study suffers from a number of limitations. The validity of the findings would clearly have been enhanced if we were able to study a larger cohort. Also, the inclusion of patients with alcohol abuse/dependence, serious physical illness and history of psychosis may have yielded different results. 


\section{Conclusion}

PTSD is under-diagnosed, even in a tertiary, inpatient setting and almost half of co-morbid cases are missed with standard assessments. Patients with PTSD were found to be more likely to admit to using cannabis. Current standard assessments at intake may not be structured to elicit symptoms and signs of PTSD adequately. Better screening-instruments for PTSD that are more user-friendly than the CAPS should be introduced in clinical practice. Education of mental health care professionals is essential to maintain a higher level of awareness of the possibility of PTSD in all patients, so that it may be diagnosed and treated early and effectively.

\section{References}

1. Ballenger JC. Introduction - Focus on posttraumatic stress disorder. Journal of Clinical Psychiatry 2000; 61:3.

2. American Psychiatric Association. Diagnostic and Statistical Manual of Mental Disorders. IV ed. Washington: American Psychiatric Association, 1994.

3. Perkonigg A, Kessler RC, Storz S, Wittchen HU. Traumatic events and post-traumatic stress disorder in the community: prevalence, risk factors and comorbidity. Acta Psychiatrica Scandinavica 2000; 101(1):46-59.

4. Brewin CR, Andrews B, Valentine JD. Meta-analysis of risk factors for posttraumatic stress disorder in trauma-exposed adults. Journal of Consulting and Clinical Psychology 2000; 68(5):748-766.

5. Paris J. Predispositions, personality traits, and posttraumatic stress disorder. Harvard Review of Psychiatry 2000; 8(4):175-183.

6. Ballenger JC, Davidson JRT, Lecrubier Y, Nutt DJ, Foa EB, Kessler RC et al. Consensus statement on posttraumatic stress disorder from the international consensus group on depression and anxiety. Journal of Clinical Psychiatry 2000; 61:60-66.

7. Grinage BD. Diagnosis and management of post-traumatic stress disorder. American Family Physician 2003; 68(12):2401-2408.

8. Lecrubier Y. Posttraumatic stress disorder in primary care: A hidden diagnosis. Journal of Clinical Psychiatry 2004; 65:49-54.

9. Kessler RC, Sonnega A, Bromet E, Hughes M, Nelson CB. Posttraumatic-Stress-Disorder in the National Comorbidity Survey. Archives of General Psychiatry 1995; 52(12):1048-1060.

10. Bisson J, Andrew M. Psychological treatment of post-traumatic stress disorder (PTSD) - art. no. CD003388.pub2. Cochrane Database of Systematic Reviews 2005; (2).

11. Bisson JI. Post-traumatic stress disorder. British Medical Journal 2007;
334(7597):789-793.

12. Tucker WM. How to include the trauma history in the diagnosis and treatment of psychiatric inpatients. Psychiatric Quarterly 2002; 73(2):135-144.

13. Brady KT, Killeen TK, Brewerton T, Lucerini S. Comorbidity of psychiatric disorders and posttraumatic stress disorder. Journal of Clinical Psychiatry 2000; 61:22-32.

14. Nader KO, Kriegler JA, Blake DD. Clinician Administered PTSD Scale for Children and Adolescents for (DSM-IV) (CAPS-CA), Current and Lifetime Diagnostic Version, and Instruction Manual. A National Center for PTSD and UCLA Trauma PSychiatry Program collaboration. 1996. Ref Type: Report.

15. First MB, Spitzer RL, Gibbon M, Williams JBW. Structured Clinical Interview for DSM-IV Axis I Disorders, Patient Edition (SCID-P), version 2. New York: New York State Psychiatric Institute, Biometrics Research, 1994.

16. Oquendo MA, Friend JM, Halberstam B, Brodsky BS, Burke AK, Grunebaum MF et al. Association of comorbid posttraumatic stress disorder and major depression with greater risk for suicidal behavior. American Journal of Psychiatry 2003; 160(3):580-582.

17. McFarlane AC, Bookless C, Air T. Posttraumatic stress disorder in a general psychiatric inpatient population. Journal of Traumatic Stress 2001; 14(4):633-645.

18. Ballenger JC, Davidson JRT, Lecrubier Y, Nutt DJ, Marshall RD, Nemeroff $C B$ et al. Consensus statement update on posttraumatic stress disorder from the International Consensus Group on depression and anxiety. Journal of Clinical Psychiatry 2004; 65:5562.

19. Seedat S, Nyamai C, Njenga F, Vythilingum B, Stein DJ. Trauma exposure and post-traumatic stress symptoms in urban African schools - Survey in Cape Town and Nairobi. British Journal of Psychiatry 2004; 184:169-175.

20. Carey PD, Stein DJ, Zungu-Dirwayi N, Seedat S. Trauma and posttraumatic stress disorder in an urban Xhosa primary care population: Prevalence, comorbidity, and service use patterns. Journal of Nervous and Mental Disease 2003; 191 (4):230-236.

21. Johns A. Psychiatric effects of cannabis. British Journal of Psychiatry 2001; 178:116-122.

22. Gray MJ, Litz BT, Hsu JL, Lombardo TW. Psychometric properties of the life events checklist. Assessment 2004; 11 (4):330-341.

23. Vaishnavi S, Payne V, Connor K, Davidson JRT. A comparison of the sprint and caps assessment scales for posttraumatic stress disorder. Depression and Anxiety 2006; 23(7):437-440. 